\author{
Military Technical College \\ Kobry El-Kobbah, \\ Cairo, Egypt
}

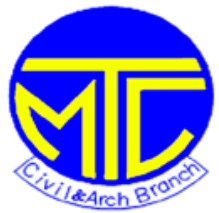

\author{
$11^{\text {th }}$ International Conference \\ on Civil and Architecture \\ Engineering \\ ICCAE-11-2016
}

\title{
CHANGE DETECTION FROM AIRBORNE LIDAR DATA FOR URBAN AREA
}

\author{
Hassan Elsaid Elhifnawy \\ Civil Engineering Department, Military Technical College, Cairo, Egypt
}

KEY WORDS: LIDAR, Point Cloud, LIDAR Images, 3D Representation

\begin{abstract}
:
The last few years Light Detection And Ranging (LIDAR) system is widely used for generating digital terrain models. LIDAR system outputs are more accurate, have higher density, are produced more rapidly, and with acquired less expensively. The developments of LIDAR systems have many of the elements that meet these properties. Airborne LIDAR has become commercially used for many environmental, engineering and civil applications, and can provide accurate data for topographic surfaces and non-terrain objects.

Changes on urban area may be happened due to city development or effect of environmental hazards. LIDAR data for an urban area is used for producing two height images, before and after changing. Search technique is applied to detect the area of changes and the objects in this area. LIDAT data with rich spatial information is recommended to be the most suitable data for change detection applications on urban areas.
\end{abstract}

\section{INTRODUCTION}

LIDAR data provides full spatial information for ground and non-ground objects, so it produces significant positioning information for all features. LIDAR systems provide not only positioning information but also property information, referred to as intensity values, for all scanned points. Two types of images can be produced from this information, a range image and an intensity image. A range image is based on height values while an intensity image is based on the intensity values for all scanned points [Shan and Toth, 2009]. A LIDAR system provides dense positioning information for surface topography and all features for the study area. LIDAR system provides not only positioning information but also property information that is called intensity values for all scanned points. The pixel values in the intensity image are calculated based on the transmitted and received energy of the laser pulse. These values depend on the object material and its efficiency for absorbing laser energy [Vain et al., 2010].Two types of images can be produced from this information, a range image and an intensity image. Range image is based on height values, while intensity image is based on intensity values for all scanned points [Al-Durgham, 2007]. LIDAR system is composed of many sensors to get this information with high density and accuracy. This 
paper introduces an airborne LIDAR system components and their work flow to end up with LIDAR data. This research tests the efficiency of using LIDAR data concentrating of height information in detecting changes in urban areas.

\section{LIDAR SYSTEM COMPONENTS}

A LIDAR system consists of two main components, a collecting information component and a navigation component. Laser scanner is the main sensor for collecting, as it collects both dense range and intensity information for the scanned area [Al-Durgham, 2007; El-Sheimy et al., 2005; Habib et al., 2009]. Developed productions of LIDAR system contain additional collecting information sensors such as a digital imaging sensor. These sensors collect not only positional information but also semantic information for each scanned point. Global Positioning System (GPS) and Inertial Navigation System (INS) are the navigation components is composed of the systems. Figure 1 shows the principal components of LIDAR system [Eid, 2011].

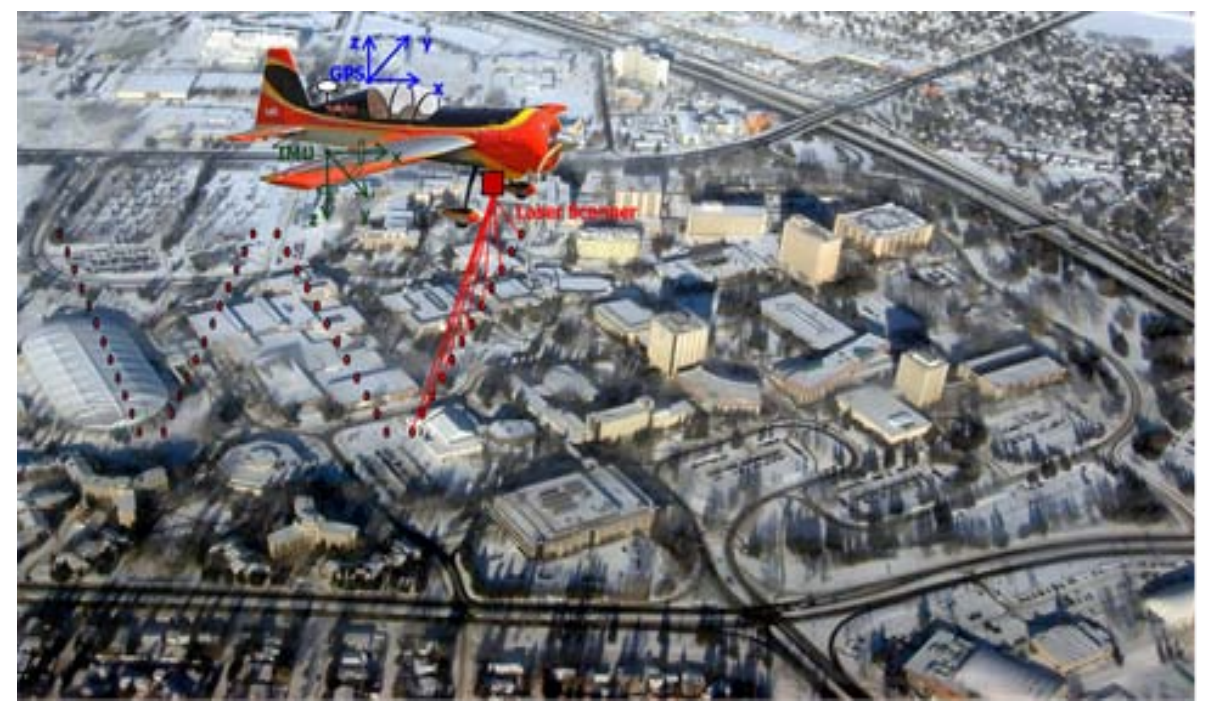

Figure 1: LIDAR System Components

\subsection{Data Acquisition}

The laser scanner contains a rotating mirror that reflects the laser beam and produce required scanning pattern. Laser scanner may contain one mirror; in this case, the scanning pattern can be produced as linear, zigzag, or nutating. The scanner pattern takes ellipsoidal shape when laser scanner contains two rotating mirrors. Linear scanning pattern (Figure 2(a)) is not preferable because there is no data collected for areas between scanned lines. Zigzag scanning pattern (Figure 2(b)) provides continuous collecting data for scanning area. Nutating scanning pattern (Figure 2(c)) provides dense collecting data with low flying speed. Elliptical scanning pattern (Figure 2(d)) is preferable because it gives dense collecting data with any speed since it makes duplication of collecting data for scanned area but with more complicated calculations for sensor modelling due to existing of two mirrors that rotating in different scanning angles [Eid, 2011; El-Sheimy et al., 2005]. 


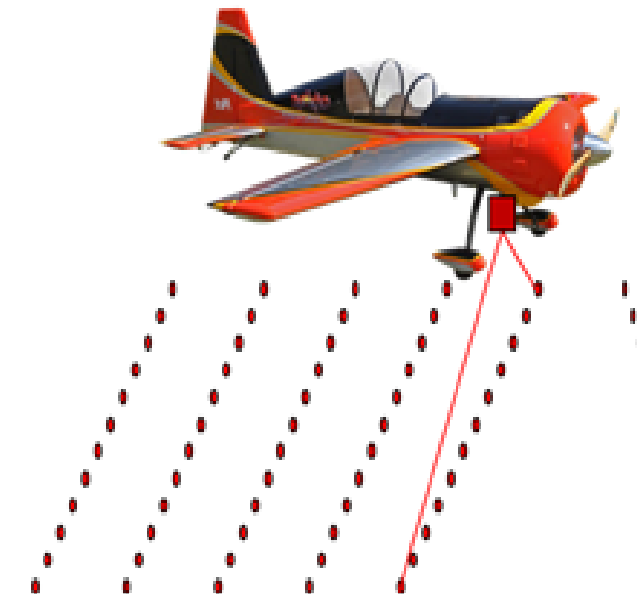

(a)

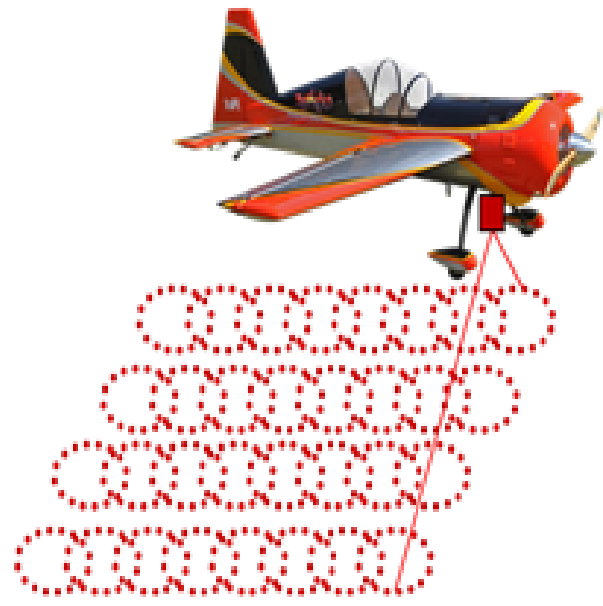

(c)

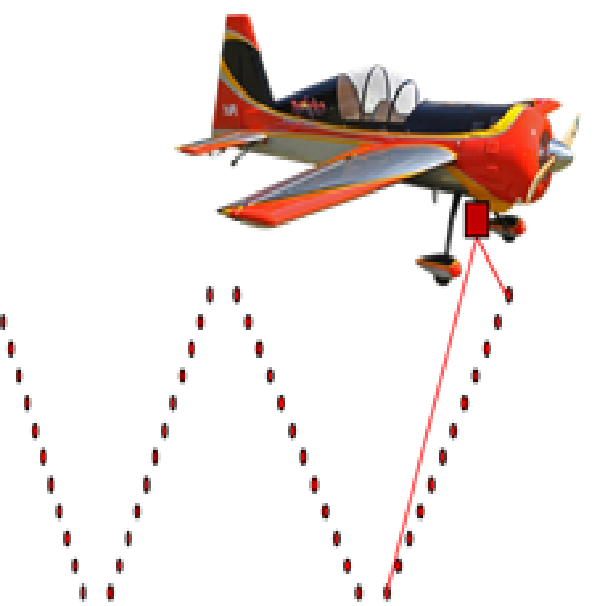

(b)

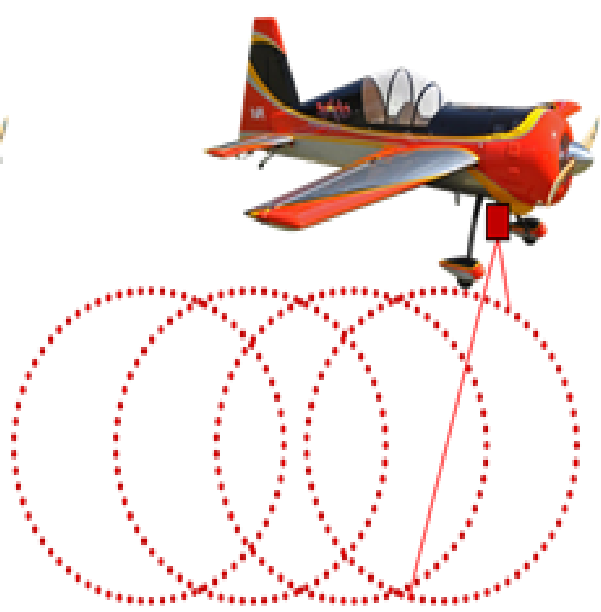

(d)

Figure 2: LIDAR Scanning Systems [Eid, 2011]

\subsection{Data Processing}

The work flow of the collecting data are as follows: Inertial Measurement unit (IMU) collects navigation data for flying mission, a GPS antenna collects coordinates of flying directions referenced to the GPS coordinate system, and laser scanner collects range and intensity information for each scanned point. IMU and GPS are used to get the trajectory for the flying direction referenced to ground coordinate system. The trajectory coordinates are used with LIDAR system calibration parameters and range information to calculate ground coordinates for each point. A LIDAR system is a direct georeferencing system, so the ground coordinates of point of interest are calculated as a vector summation as shown in Figure 3 that represents a LIDAR equation [Eid, 2011; El-Sheimy et al., 2005]. Equation 1 [Habib et al., 2009] represents a mathematical model of LIDAR equation that is used for calculating ground coordinate of each scanned point. 


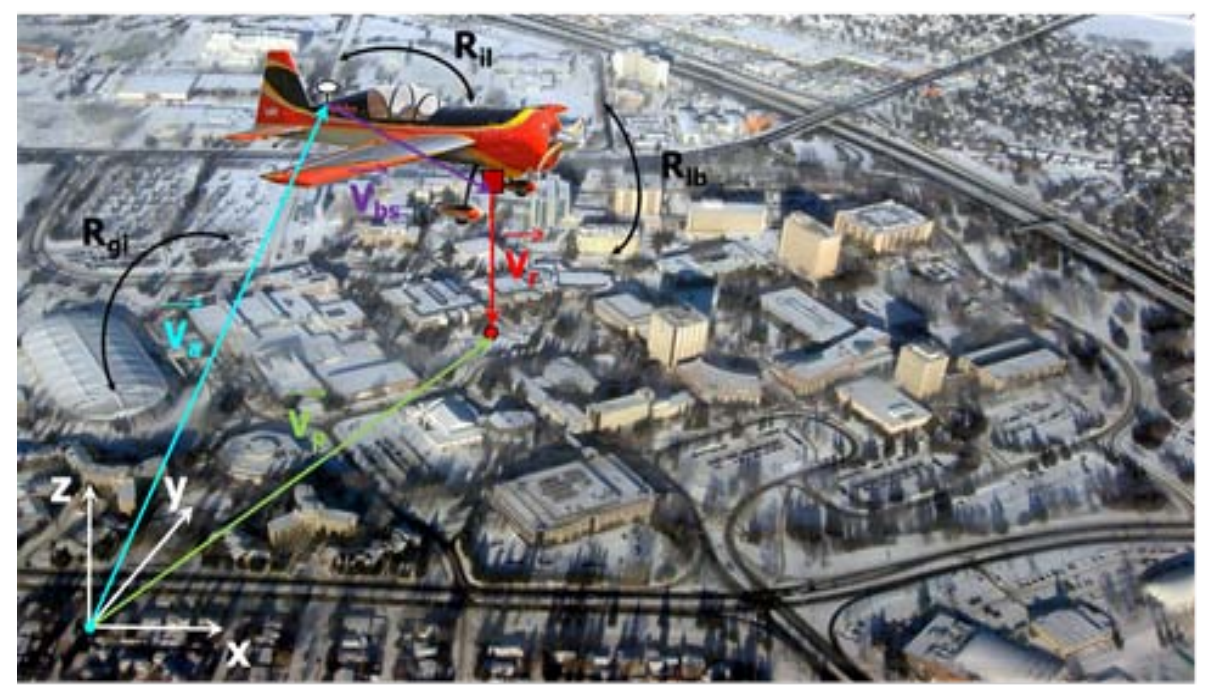

Figure 3: LIDAR Coordinate System [Eid, 2011]

$\overrightarrow{V_{p}}=\overrightarrow{V_{a}}+R_{g i} \overrightarrow{V_{b s}}+R_{g i} R_{i \mathbb{i l}} R_{\mathbb{U} b} \overrightarrow{V_{Y}} \quad 1$ [Habib et al., 2009]

where: $\quad \overrightarrow{V_{p}}=$ Ground coordinates of point of interest

$\overrightarrow{V_{\alpha}}=$ Ground Coordinates of antenna phase center

$\overline{V_{b}}=$ Vector of lever arm between antenna phase center and center of laser scanner coordinate system with respect to IMU coordinate system

$\vec{V}_{r}=$ Vector of range of point of interest with respect to laser beam coordinate system

$R_{g}=$ Rotation matrix between IMU coordinate system and ground coordinate

$R_{\tilde{\mathrm{I}}}=$ Rotation matrix between IMU coordinate system and laser scanner coordinate system

$R_{\mathbb{1}}=$ Rotation matrix between laser scanner coordinate and laser beam coordinate system

Positional, spatial and intensity information are irregular data form a point cloud. This data has to be redistributed on a grid using an interpolation technique to produce LIDAR images, a range image and an intensity image. The grid is same for both images but for a range image the pixel values represent the height of scanned points, while for an intensity image the pixel values represent intensity values [Al-Durgham, 2007]. Figure 4 shows a sample for range (height) image and an intensity image for same area study.

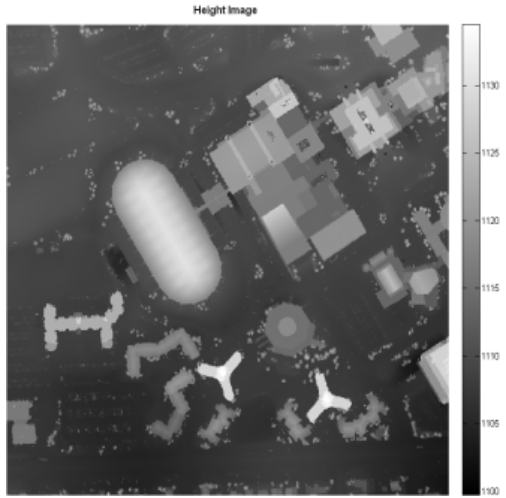

(a) (Rangle Image)

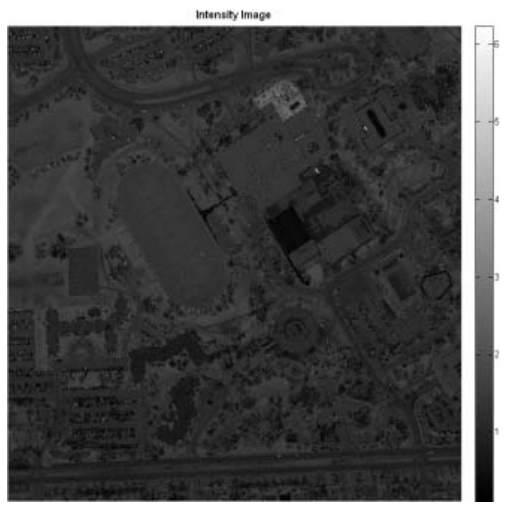

(b) (Intensity Image)

Figure 4: LIDAR Images 


\section{RESEARCH ALGORITHM}

Image of area of study is produced by applying certain interpolation technique on the raw LIDAR data for this area. Raw LIDAR data for a part of area of study is extracted from the original raw LIDAR data for the area of study. The modification in $\mathrm{z}$ value are applied on all points of the extracted LIDAR data. Raw LIDAR data for the area of study is investigated to represent as another LIDAR data for area of study after modifications (Changed area of study).

Two images for are produced by using same interpolation technique, one for area of study without modifications and another after modifications. These two images produced from same interpolation technique with same sampling distance are considered as an input images for a case of study. Detection of changes are detected after applying a certain search algorithm through MATLAB environment and produce an image of change. Figure 5 shows the schematic diagram for the work flow that is applied to prepare the case of study in the research.

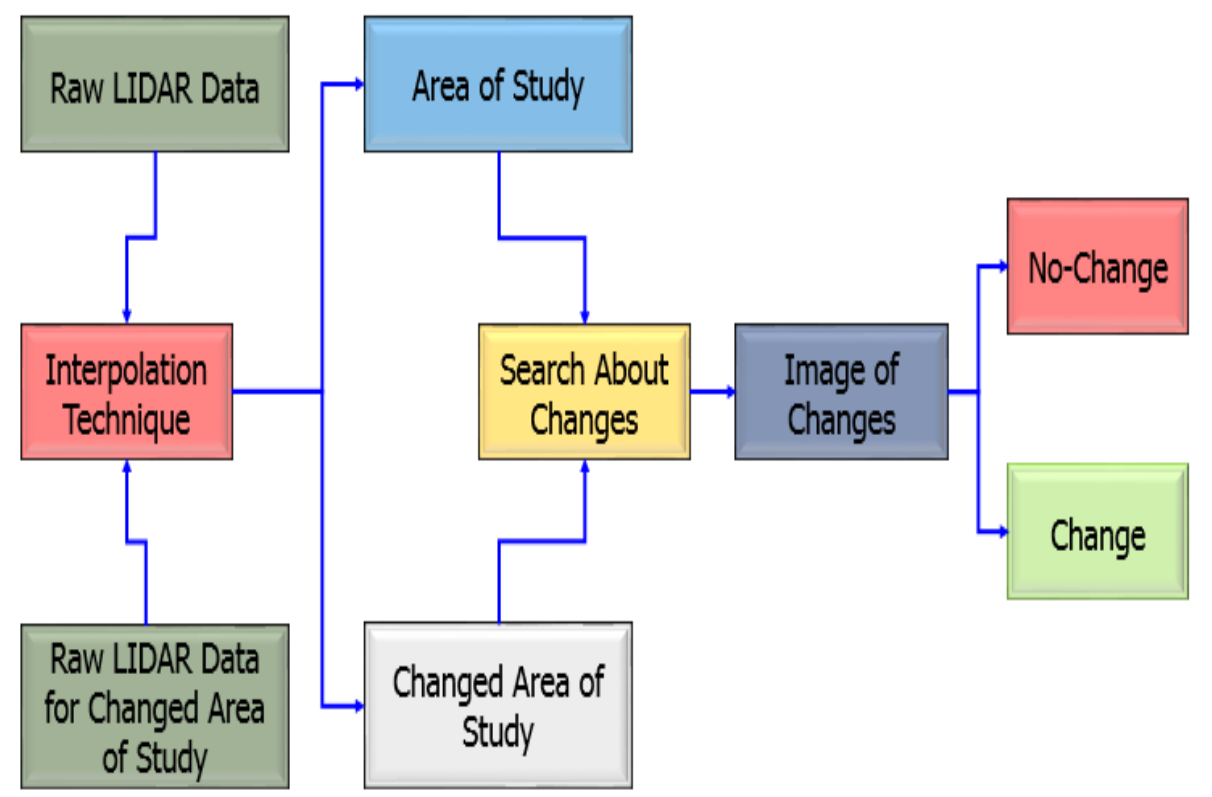

Figure 5: Work Flow of Preparing Data for Research

\section{LIDAR DATA FOR AREA OF STUDY}

Since the main target of this research is to introduce the capability of a LIDAR system to represent both on terrain and off terrain objects with dense spatial information, then the selection of the study area was concentrated on the region urban area that contains buildings, roads and trees. The selected area of study has to be focused on a region rich with buildings to represent an urban area. This condition is provided by the selected area of study that is located in Calgary, Canada. The area of study was scanned by Optech ALTM 3100 LIDAR system (Figure 6). The LIDAR system scanned this area with a zigzag scanning system with a total amount of scanned points equalling 374,746 points. 


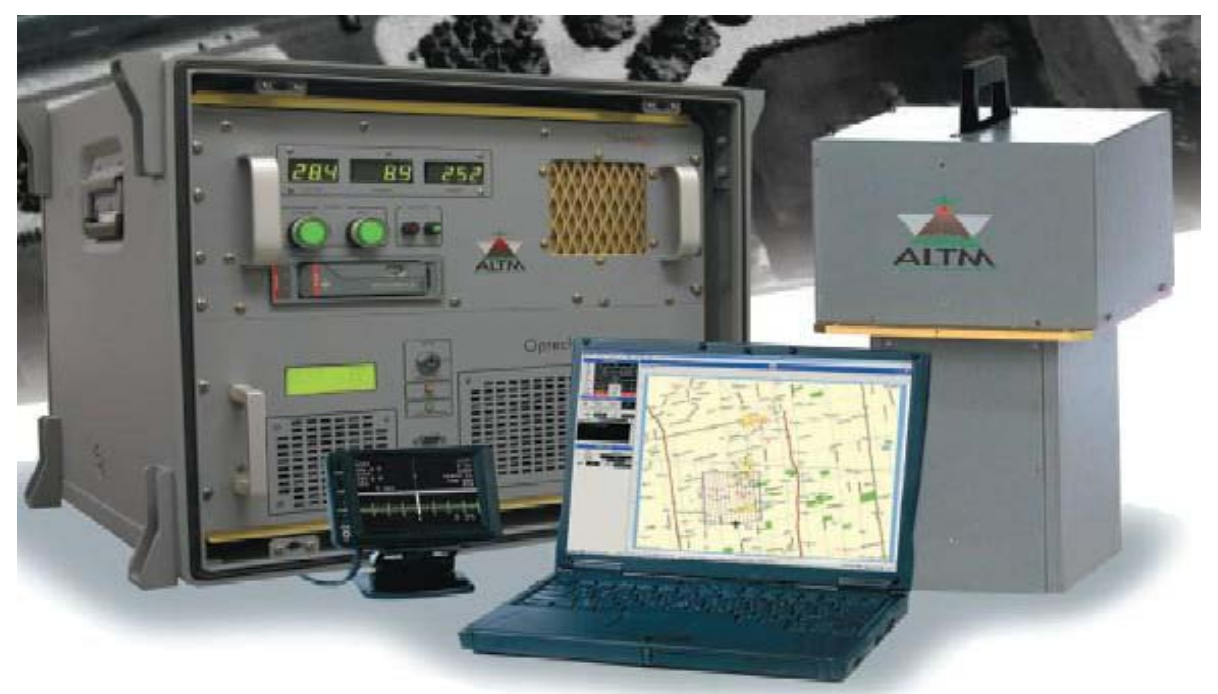

Figure 6: Optic ALTM LIDAR System [Al-Durgham, 2007]

\subsection{LIDAR Point Clouds}

The LIDAR data of an area of study can be represented as a point cloud. The data contains dense positioning information for each scanned point. Figures (Figure 7 and Figure 8) shows a LIDAR point cloud for an area of study base on height information from two different sighting angles.

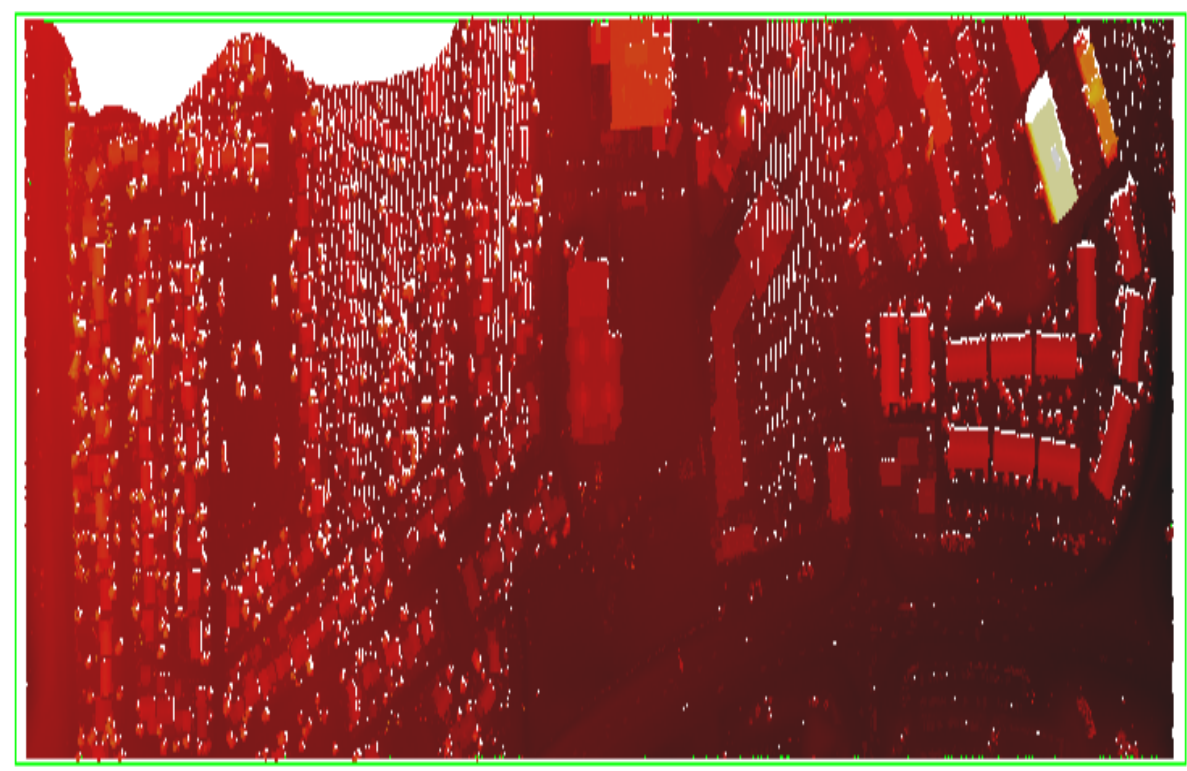

Figure 7: Nadir LIDAR Point Cloud 


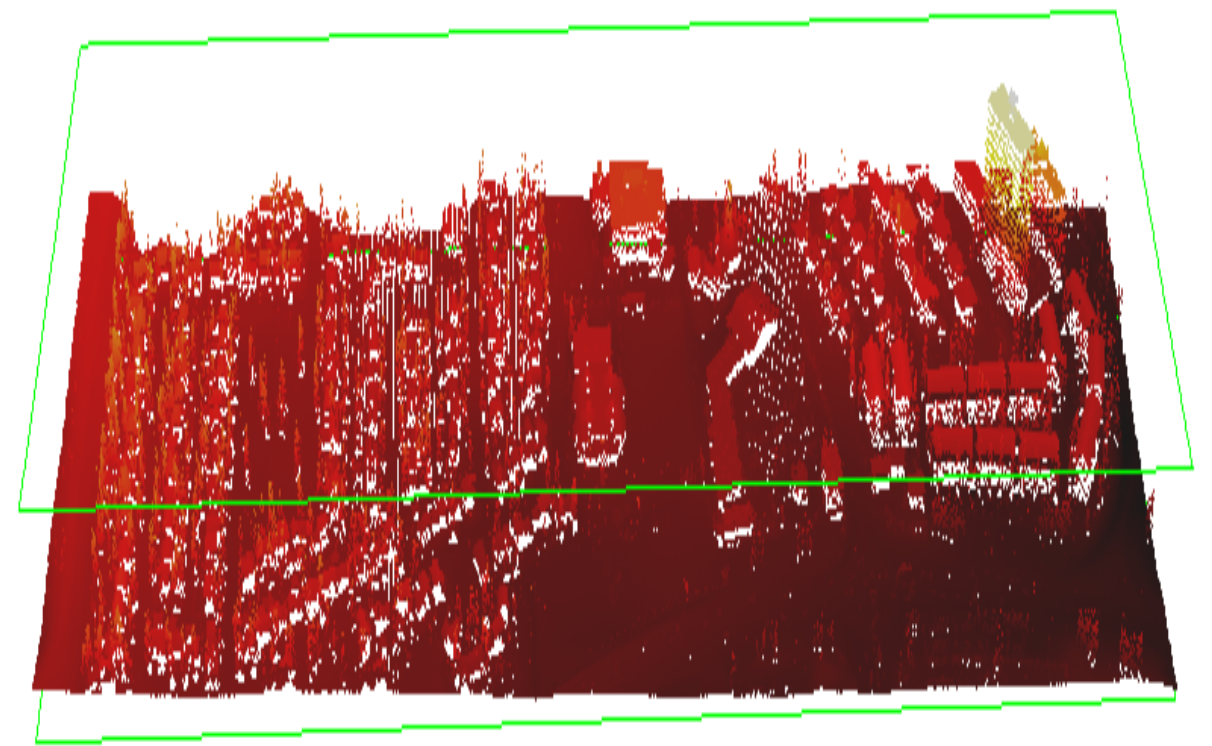

Figure 8: Oblique LIDAR Point Cloud

\subsection{LIDAR Images}

The distribution of scanned points is irregular, it is necessary to regularize this distribution to produce LIDAR images (height and intensity). Redistribution LIDAR point cloud is applied by using interpolation technique to generate equally spaced mesh and calculate height and intensity information for each grid node. Figures (Figure 9 and Figure 10) show the height and intensity images for the area of study after applying an interpolation technique over $0.25 \mathrm{~m}$ equally spaced mesh.

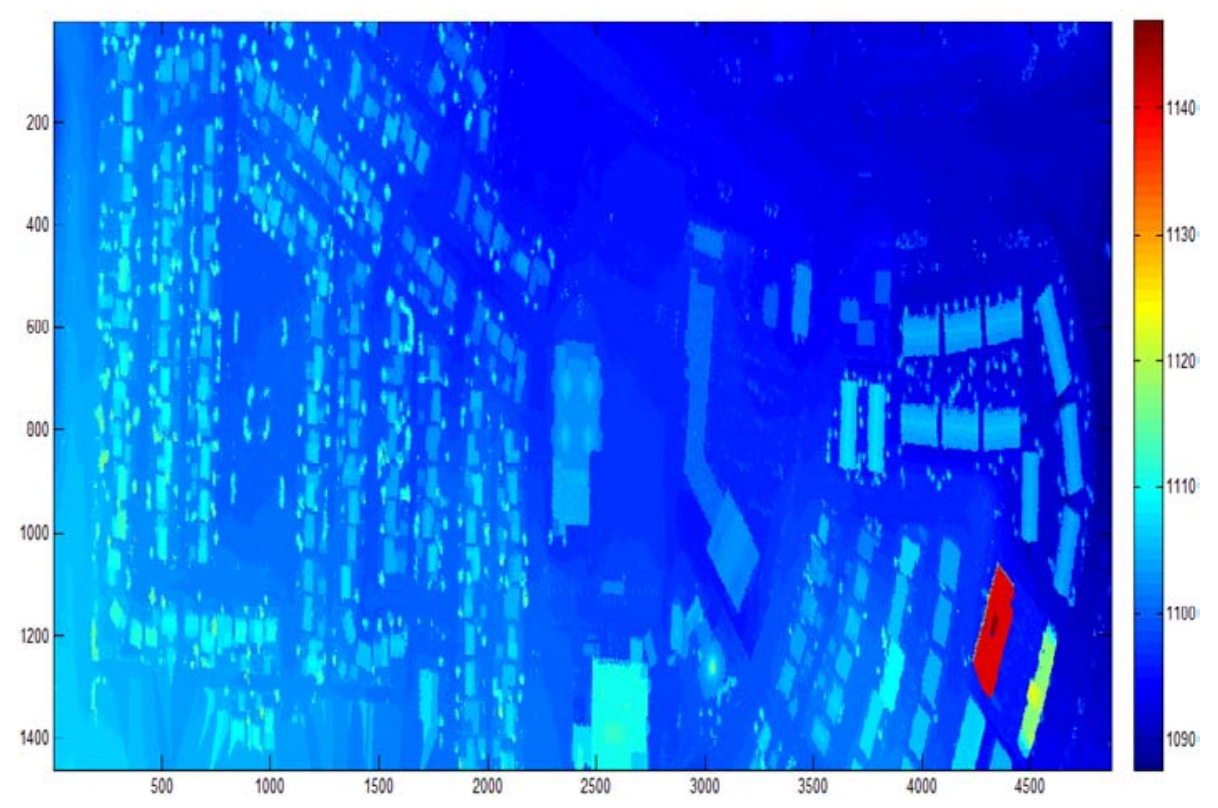

Figure 9: Height Image 


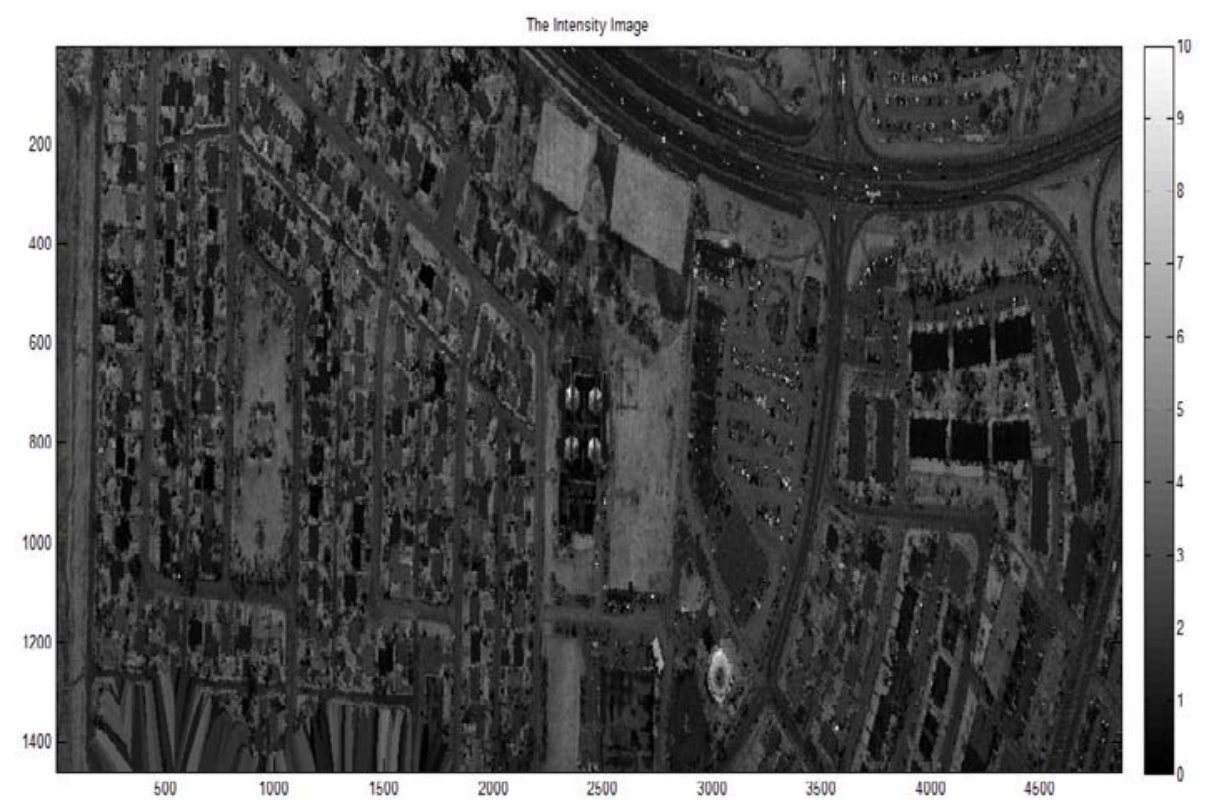

Figure 10: Intensity Image

\subsection{D Representations and Models}

Height image is considered as Digital Surface Model (DSM) for an area of study. DSM can be represented in 3D for viewing and magnifying the topographic properties for all scanned feature as shown in Figure 11.

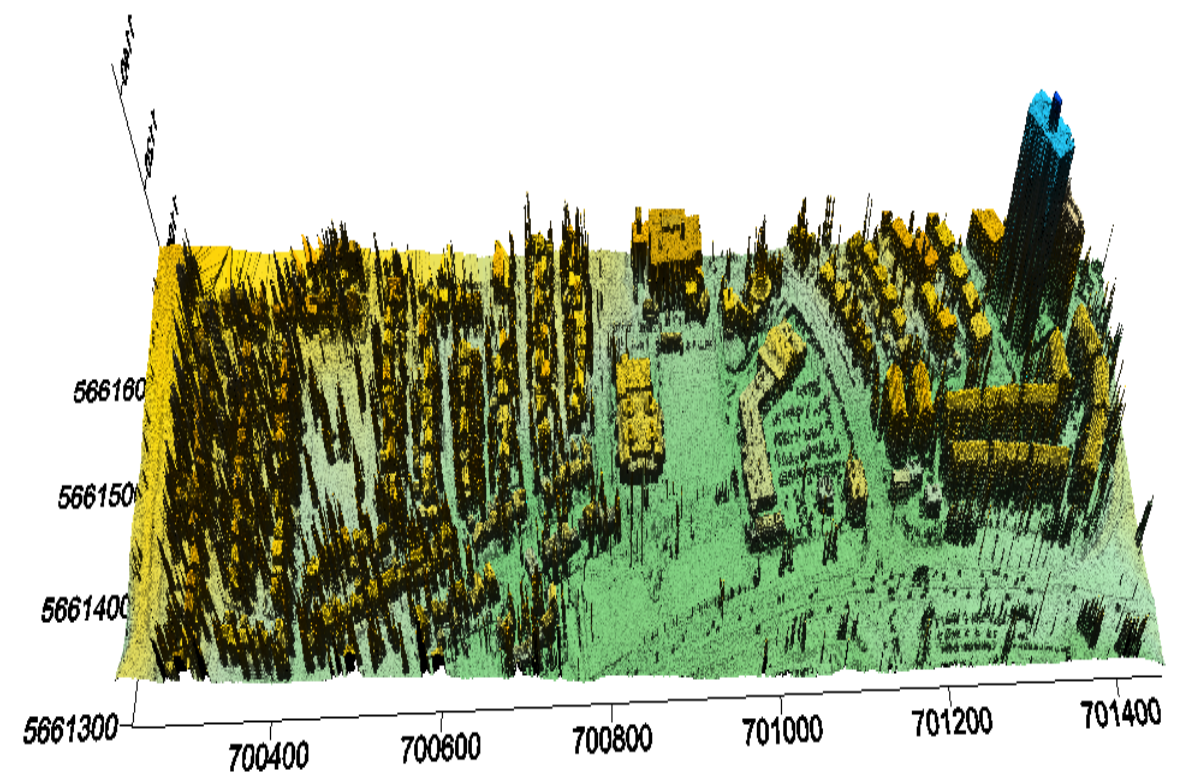

Figure 11: 3D Object Representation for DSM

\section{CHANGE DETECTION AND COMPARATIVE STUDY}

A part of the raw data for the area of study bounded by min x-coordinates equal to $700706.54 \mathrm{~m}$ and max x-coordinates equal to $700877.13 \mathrm{~m}$ and min y-coordinates equal to $5661430.6 \mathrm{~m}$ and max y-coordinates equal to $5661553 \mathrm{~m}$ is selected to be changed area. The height values of all raw points of this area are modified. Figures (12 and 13) show the height image of area of study after changing and the 3D representation respectively. 


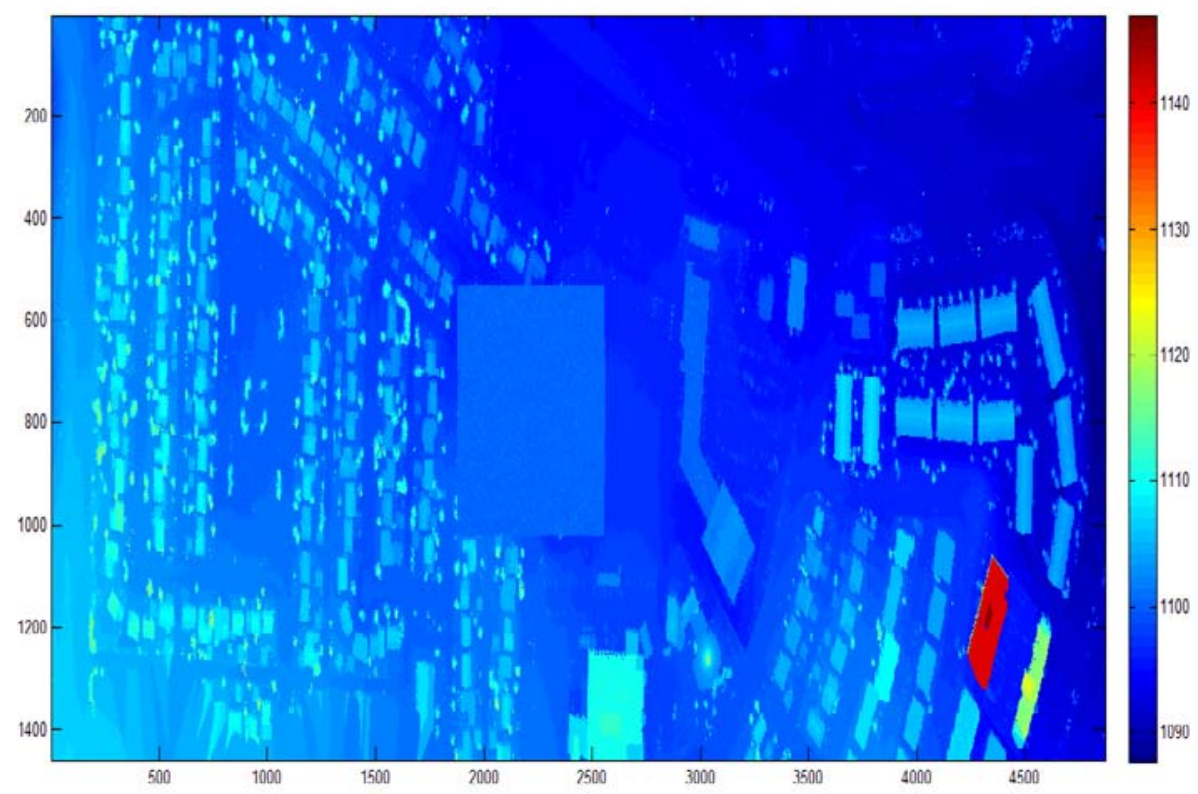

Figure 14: Height Image after Changed

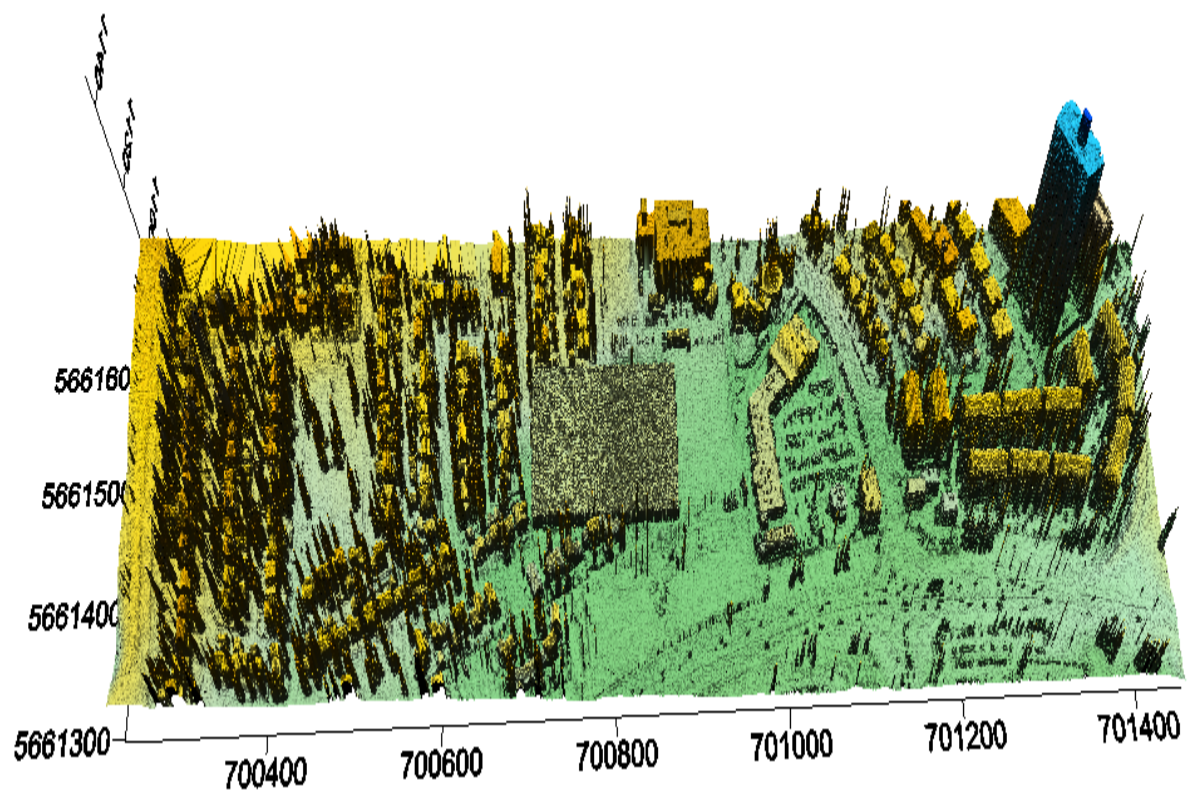

Figure 15: 3D Representation after Changed

\subsection{Change Detection Results}

The change detection results are represented on the change image. This image shows the locations of pixels that are affected by the modifying height values of the changed area. This image contains only two colors, the red color which represents the pixels of no changes and the green color which represents the pixels that have changes. Figure 16 shows the change images for the area of study. 


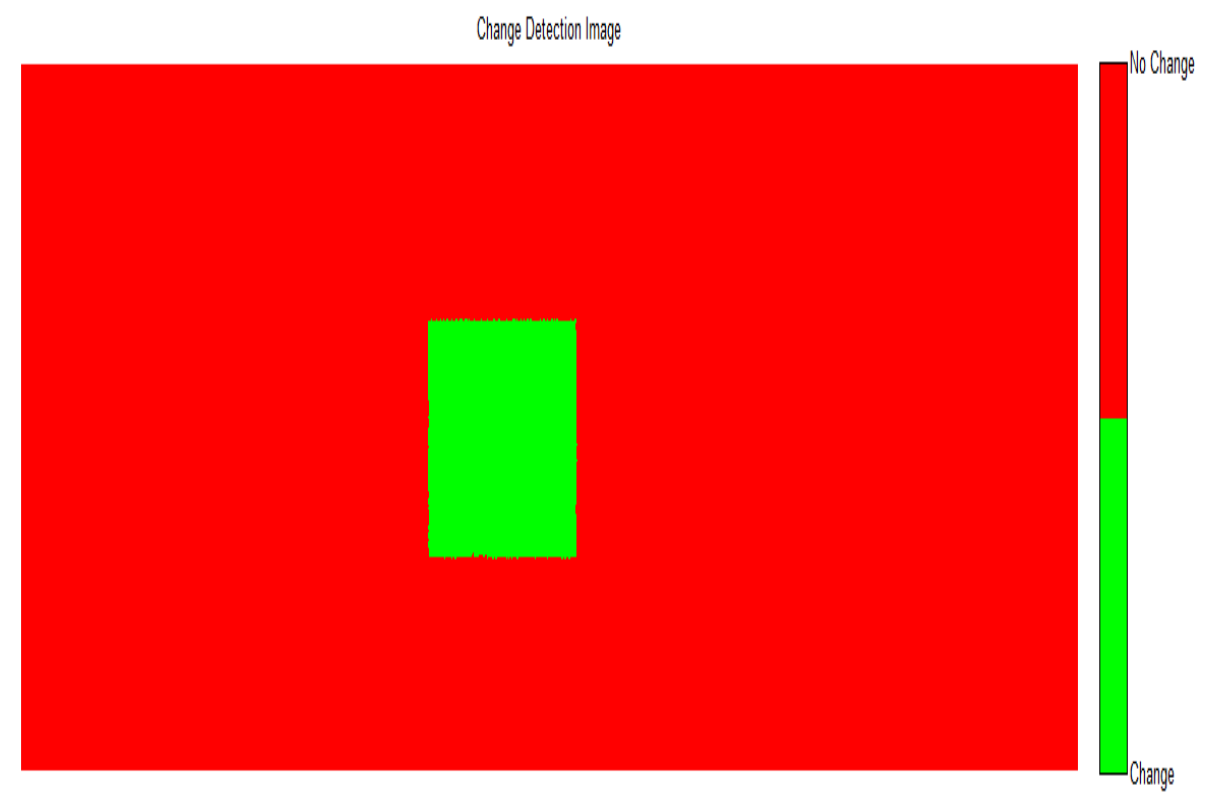

Figure 17: Change Detection Image

\subsection{Images and 3D Representation of Detected Changes}

The objects that cause the changes in the input image have to be identified based on the applications, for example, city development or environmental hazards effects. The objects can be identified by producing images of changed area using the extracted raw LIDAR data of the detected changed area. Objects in the changed area can be identified visually in by producing 3D representation for the changed area. Figures (18 and19) show the image of changed area and the 3D representation of the detected objects respectively.

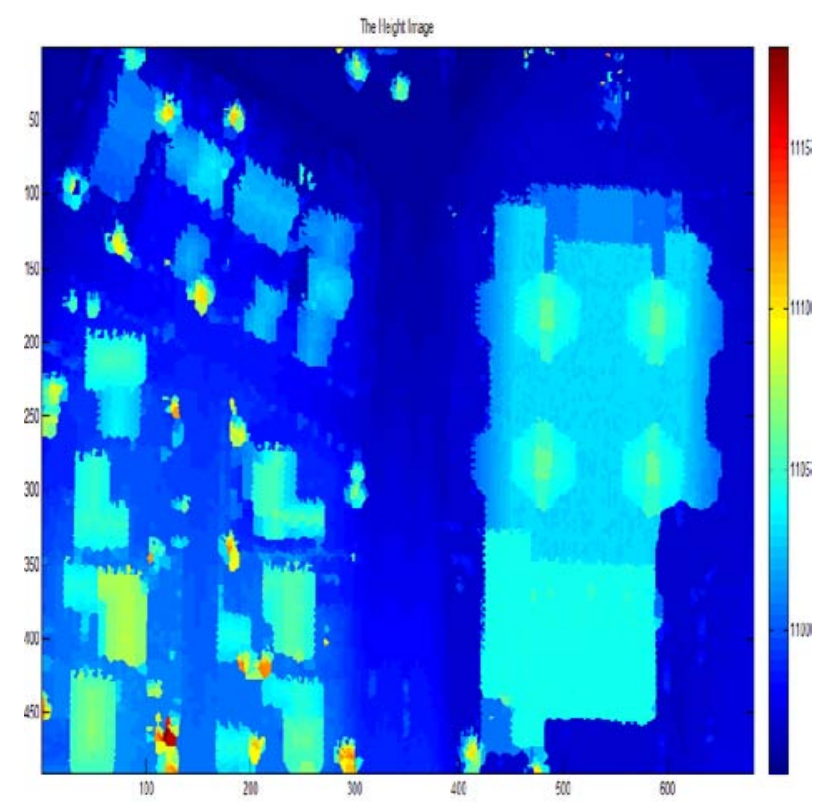

Figure 20: Image of Area of Change 


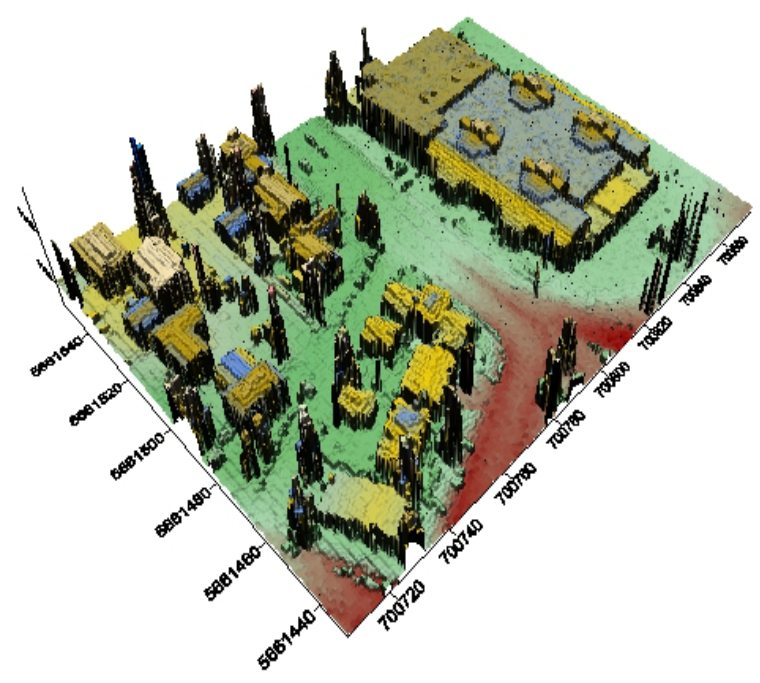

Figure 21: 3D Representation for Area of Change

\section{SUMMARY, CONCLUSIONS, AND FUTURE WORK}

Airborne LIDAR system has a great capabilities to get a dense and accurate spatial information for on terrain and off terrain objects. LIDAR system provides not only height information but also intensity information that can be used as a semantic properties for scanned features. LIDAR data is produced as an irregular point cloud that contains spatial and intensity information. LIDAR point cloud is used to generate two different outputs in a regular form as image (height and intensity images). Height image is a DSM that contains elevations for all scanned objects. DSM is represented in 3D to show the topography of all features in the area of study.

Airborne LIDAR system has to be the main source for DSM of any areas of interests. Due to the properties of outputs from airborne LIDAR system, it can be considered as a powerful standalone tool that can be used for detecting changes with high accuracy for all types of areas. LIDAR data is considered as an efficient source for detecting off terrain objects even they are camouflaged. LIDAR data with other from imaging sensor can provide dense spatial and semantic information that will be used for many civil and military applications. LIDAR

\section{REFERENCES}

Al-Durgham, M. (2007) Alternative methodologies for the quality control of LiDAR systems, M.Sc., University of Calgary (Canada), Canada

Eid, H. (2011) "Automated urban features classification and recognition from combined RGB/lidar data,"

El-Sheimy, N., C. Valeo, and A. Habib (2005) Digital terrain modeling : acquisition, manipulation, and applications, Artech House, Boston

Habib, A., K. I. Bang, A. P. Kersting, and D. C. Lee (2009) "Error Budget of Lidar Systems and Quality Control of the Derived Data," Photogrammetric Engineering and Remote Sensing, vol 75, no 9, Sep, pp. 1093-1108 
Shan, J., and C. K. Toth (2009) Topographic laser ranging and scanning: principles and processing, CRC Press/Taylor \& Francis Group

Vain, A., Y. Xiaowei, S. Kaasalainen, and J. Hyyppa (2010) "Correcting Airborne Laser Scanning Intensity Data for Automatic Gain Control Effect," Geoscience and Remote Sensing Letters, IEEE, vol 7, no 3, July, 2010, pp. 511-514 